\title{
Fashion Android Application using Augmented Reality
}

\author{
Soundarya. S. Jetty ${ }^{1}$, Akshatha. R. $\mathrm{P}^{1}$, Pooja. $\mathrm{C}^{1}$, Mamatha. $\mathrm{M}^{1}$, Nagaraj. $\mathrm{A}^{2 *}$ \\ ${ }^{1}$ Under Graduate Student, ${ }^{2}$ Associate Professor, \\ Dept. of Computer Science \& Engineering \\ Jyothy Institute of Technology, Visvesvaraya Technological University Thataguni Post, \\ Bengaluru-560082, India
}

\begin{abstract}
In spite of the fact that we are in 21 st century with loads of advancement in the field of innovation and improved periods of utilizing applications inside the scope of our fingertips, the desire towards a novel and better innovation for a life of simplicity ne'er comes to stop. One such new innovation is expanded reality. This is an application where clients can shop online in the most astute manner by taking a stab at various embellishments like caps, shades, pieces of jewellery and so forth simply utilizing our versatile camera to. This application gives an easy to understand frontal picture of face with no/light cosmetics and short/bound hair. The Beauty specialist's framework won't just propose the client appropriate cosmetics and hairdo, it even shows the manufactured impacts.
\end{abstract}

Keywords - Augmented Reality (AR), Face Detection, Object Detection, Android OS, Smart Phone.

\section{INTRODUCTION}

Augmented fact era with this tread occurs attractively as interest about AR heats up. Increased fact was a favourable location those days, having a functionality to assemble the destiny platform for computing, this gives all tech companies a highly competitive environment.The concept of Augmented Reality was a combination of digital environments. It gives a daily look at digital factorsHaving a clever phone camera on a daily basis, in a mixed factual environment where each VR and AR are combined, is actually foreign and is called communicating as a digital gadget.AR truly shows the consumer objects in its natural habitat. For a number of years augmented reality has been buzzing music, but how fashion and beauty are influenced by this technology. Augmented truth is a physical's live view;

This technology has our capacity to take advantage of situations, expanding with new gadgets produced with the help of us every now and then. AR with more appealing facial control algorithm capabilities strike or identify the face and its elements say, nose, mouth, eyes, or hair and the era allows for real face alteration.

\section{LITERATURE SURVEY}

It uses a depth camera to capture the figure of a user standing in front of a large display screen.The display can show fashion concepts and various outfits to the user, coordinated to his or her body. Thus, a "magic mirror" effect is produced.They support total fashion coordination for accessories and outfits automatically, and does not require computer or fashion expertise.[1]
The customer has the benefit to shop online in a smart way by trying on different accessories like sunglasses, hat, necklaces, etc. Using webcam. This application helps the user to enter the zone of the camera, the camera will starts tracing the movements of the user with the detection algorithm working in the background.[2]

Finding the faces by colour are of two types they are Face Detection in colour image and Face Detection in colour images using PCA. Face Detection in colour image: The system relies on a two-step process which first detects regions which are likely to contain human skin in the colour image and then it extracts information from these regionswhich might indicate the location of a face in the image. [3]

The system which allows the user to interact the system for try on lipstick.User can select a colour on a colour scale and see as if she tries on this colour of lipstick. We have presented an approach to realize real-time virtual try-on of lipstick by user interaction.[4]

The system physically simulates the selected virtual clothes on the user's body in real-time which help the user to see virtual clothes fitting from multiple angles as he/she moves. They are implementing automatic virtual Try-on system. [5]

\section{WORKING}

AR Demo, is basically a shopping cart. Each Users gets registered in the application using user credentials. New users who wish to register by providing the following credentials and once successfully registered, the user can login to the Application. The user can try on accessories virtually. When the users Selects a particular item to try on, that particular item augmented camera opens and the selected item pops up and the user can virtually wear and check it out.

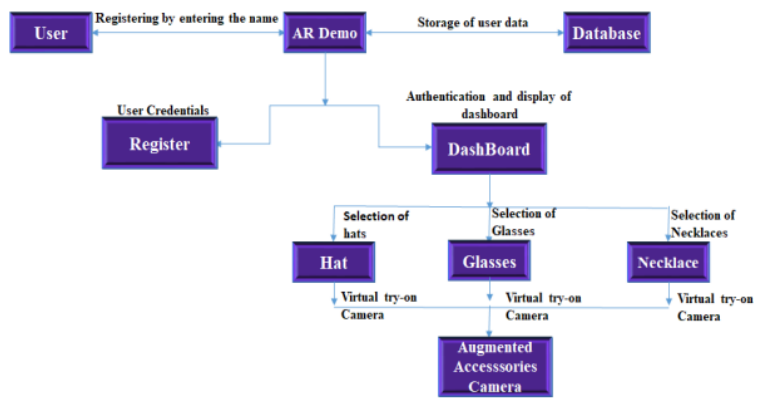

Fig 3.1: Structure chart for the working of app 
- The computer system is connected to an android device. The application would run on the Android Studio software and the application gets installed on the respective landroid device.

- After installed the application on the android device, the user then registers into the lapplication by entering the required user credentials such as first name, last name, email, phone number, and password.

- After registering to the application the user as to login by entering the email and password which he/she entered while registering.

- When the user logs in with correct user credentials the dashboard appears which as the different category of accessories the user can select any of the accessories.

- Once the user selects the accessories category the related data will be displayed from where the user can select the accessory.

- Once the user selects the accessory the camera is opened and the selected accessory is displayed and the user can virtually wear the accessories and check out weather the accessory which they want to buy suits them or not.

- Once the user is done with all the trial he/she can logout from the application, which is redirect to the login page. From which you can exit the application.

\section{IMPLEMENTATION}

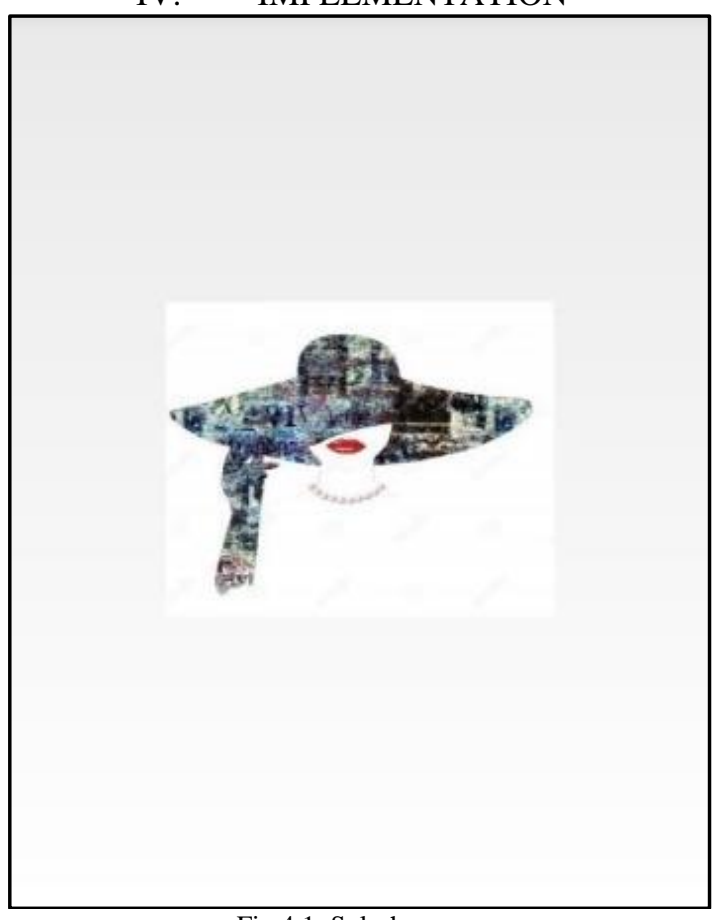

Fig 4.1: Splash page

Once the user open the 'AR Demo' app the splash page appears showing the logo of the app.

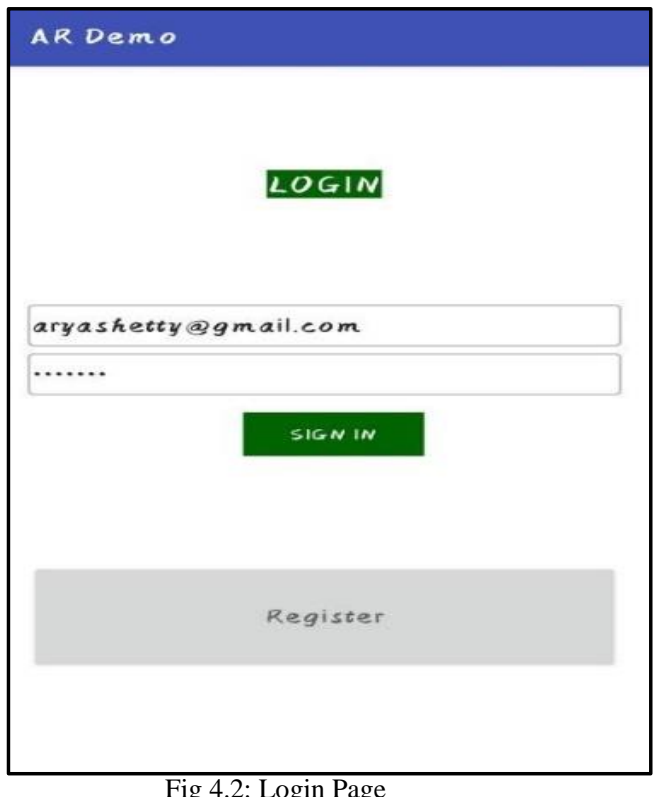

Once the user open the app splash page appears and then user has to log in by providing registered information such are email, password to try on the accessories.

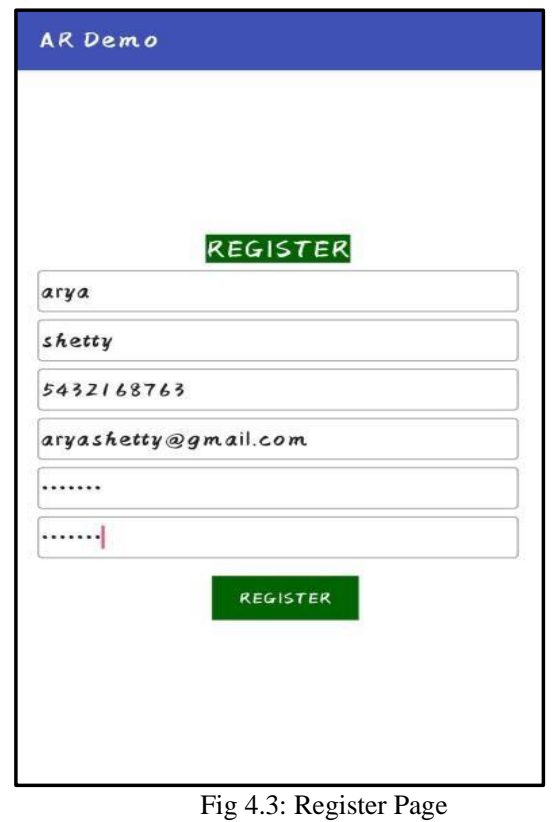

If he/she is a new to this app then they have to register by providing the required user credentials such are name, mobile number,email,password, so that they can try on the accessories. 


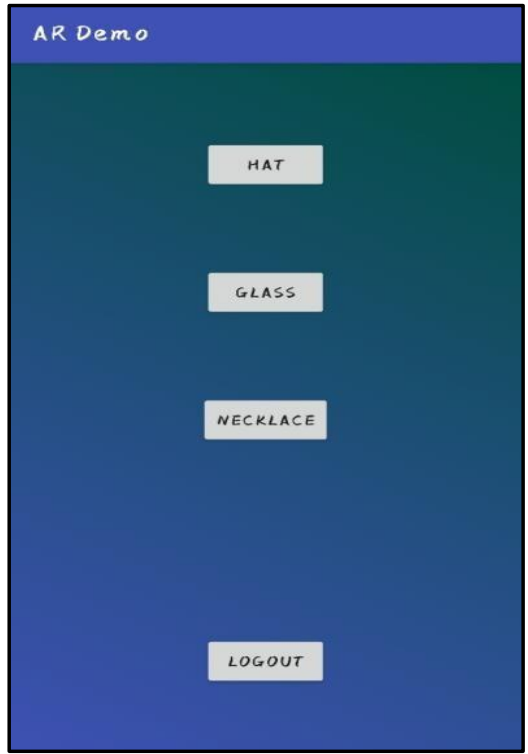

Fig 4.4 :Dashboard page

Once after the succesfull registration the dashboard paage will be displayed where the different accesssories are given such are hats, jewellery and sun glasses. Then the user has to select any one accessories which they want to try on then that particular accessories page will be displayed.
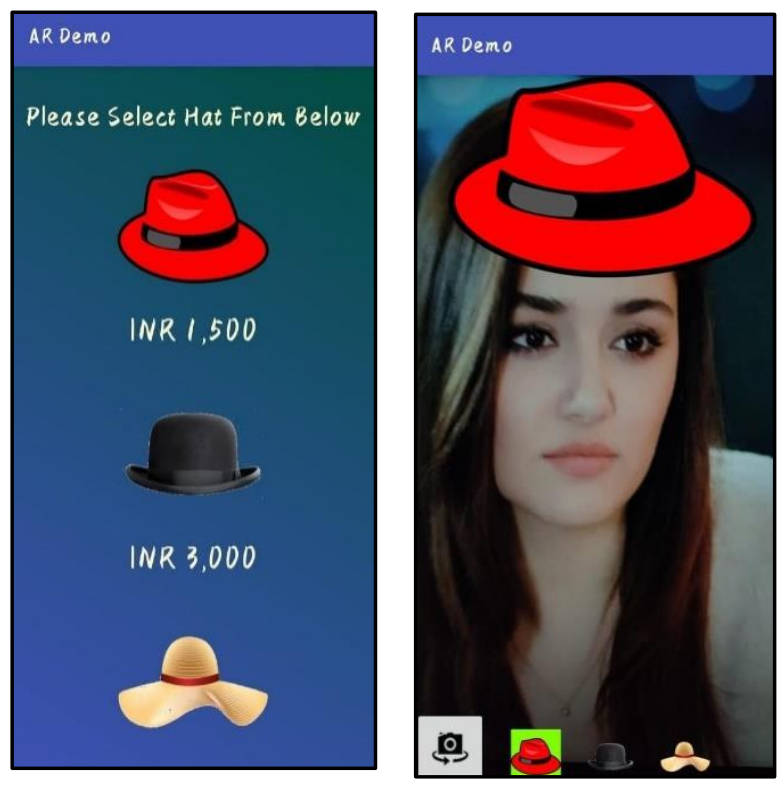

Fig 4.5: Demo of virtual hat Try-on

Here if user wants to try on the hat then he /she will have to click the button then the different types of hats will be displayed, here user can select any onehat which they want to try on virtually.

Then by clicking the particular hat, the virtual camera pop ups. And that selected item will be placed on them.

Here through which a user can know whether the accessories suits them or not. Which makes everyone to shop online easily.
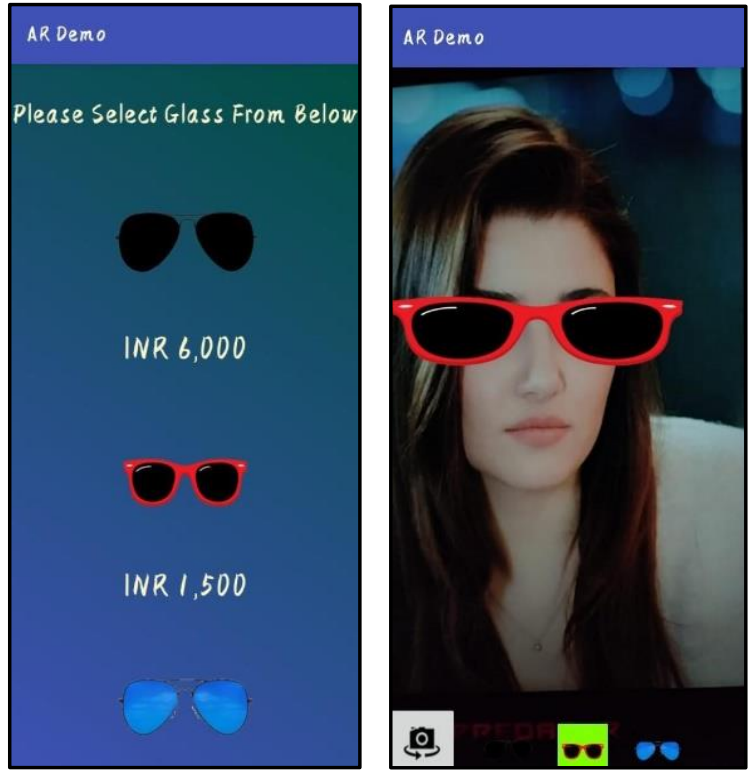

Fig 4.6: Demo of virtual Sun glasses Try-on

Here if user wants to try on the Sun Glasses then he /she will have to click the button then the different types of hats will be displayed, here user can select any one Sun Glasses which they want to try on virtually.

Then by clicking the particular sun glass, the virtual camera pop ups. And that selected item will be placed on them.

Here through which a user can know whether the accessories suits them or not. Which makes any easier to shop online.
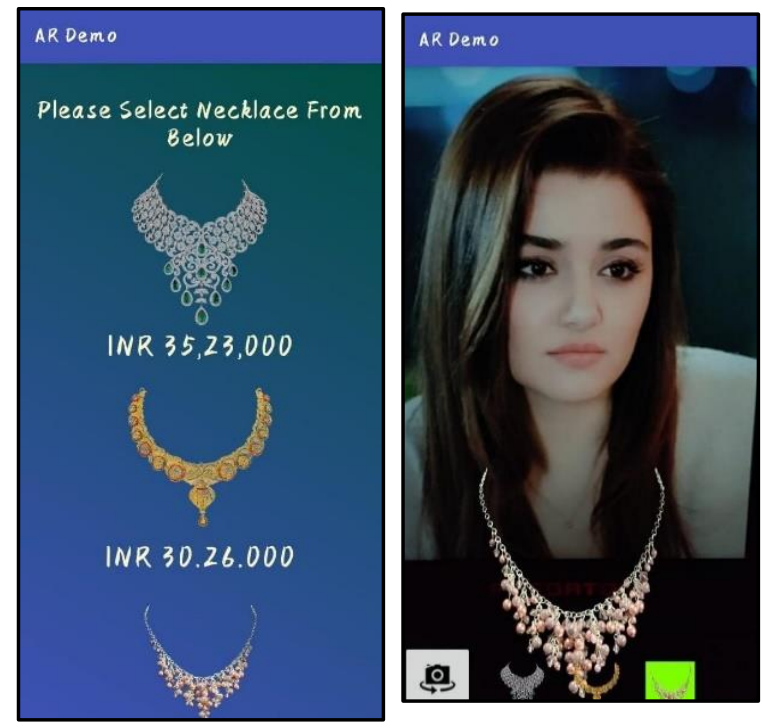

Fig 4.7: Demo of virtual JewelleryTry-on

If user wants to try on the jewellery then he /she will have to click the button then the different types of jewels will be displayed, here user can select any one necklace which they want to try on virtually. 
Then by clicking the particular necklace, the virtual camera pop ups. And that item will be placed on them.

Here through which a user can know whether the accessories suits them or not. Which makes any easier to shop online.

\section{CONCLUSION}

We know the computer has changed the way people interact. In reality this paper describes a face, and the other step is to identify the objectthrough this work plan, we add more criteria and consider the problems posed by the novel engagement with the AR that the mobile device provides. We can see a huge shift in people's behaviour, such as their muster buying, attitude, and etc.This application allows users or customers to purchase the products directly from home via internet using device or smartphone. Smart shopping fashion android uses augmented reality in this framework, applying the simplicity in costly computations and in real-time design.

\section{CONFLICT OF INTERESTS}

The authors declared no conflict of interests with respect to authorship, work and publication of this paper.

\section{ACKNOWLEDGMENT}

We authors like to acknowledge to our esteemed institution "Jyothy Institute of Technology" for providing us an opportunity and to our principal Dr. K Gopalkrishnan for providing us adequate facilities to undertake this project work. We would also like to thank Head of Department, C.S.E Dr Prabhanjan S, our family and friends who have guided and helped us through this work and preparation of this manuscript.

\section{REFERENCES}

[1] Guo, D. and Sim, T., 2009, June. Digital face makeup by example. In 2009 IEEE Conference on Computer Vision and Pattern Recognition (pp. 73-79). IEEE.

[2] Yuan, M., Khan, I.R., Farbiz, F., Yao, S., Niswar, A. and Foo, M.H. 2013. A mixed reality virtual clothes try-on system. IEEE Transactions on Multimedia, 15(8), pp.1958-1968.

[3] Kim, M. and Cheeyong, K., 2015. Augmented reality fashion apparel simulation using a magic mirror. International journal of smart home, 9(2), pp.169-178.

[4] Kachare, S., Vanga, S., Gupta, E. and Borade, J., 2015. Fashion accessories using virtual mirror. International Journal of Engineering and Computer Science, 4(4), pp.11401-11406.

[5] Oztel, G.Y. and Kazan, S., 2015. Virtual Makeup Application Using Image Processing Methods. no, 5, pp.401-404.

[6] Shaikh, A.I., Gaikwad, S.S., Bhujbal, K.M., More, S.S. and Pawar, S.E., 2016, March. Virtual Try-0N System. In National Conference "NCPCI (Vol. 2016, p. 19)

[7] Ashwitha, D. and AS, M.M., Smart Shopping Using Augmented Reality on Android OS.

[8] Köse, Ş.G. and Akgül, A.K., Innovative Approaches in Fashion Retailing. Yildiz Social Science Review, 2(2), pp.29-38.

[9] Javornik, A., Rogers, Y., Gander, D. and Moutinho, A., 2017, May. MagicFace: Stepping into character through an augmented reality mirror. In Proceedings of the 2017 CHI Conference on Human Factors in Computing Systems (pp. 4838-4849).

[10] Android shopping app using augmented reality ,"ApurvaShinde" ,Sept 2017.

[11] Oliveira, D., Guedes, P., Silva, M., Vieira e Silva, A. and Teichrieb, V., 2015. Interactive makeup tutorial using face tracking and augmented reality on mobile devices. In XVII Symposium on Virtual and Augmented Reality (pp. 220-226)

[12] El-Seoud, S.A. and Taj-Eddin, I., 2019. An Android Augmented Reality Application for Retail Fashion Shopping.

[13] Liu, L., Xing, J., Liu, S., Xu, H., Zhou, X. and Yan, S., 2014. Wow! you are so beautiful today!. ACM Transactions on Multimedia Computing, Communications, and Applications (TOMM), 11(1s), pp.1-22. 